\title{
THE SCHWARZ LEMMA AND BOUNDARY FIXED POINTS
}

\author{
MoOnJA JEONG
}

\begin{abstract}
In this note we study the Schwarz lemma and its various versions. We find a condition for a holomorphic map to have fixed points only on the boundary of the unit disc and compare its derivatives at fixed points to get some relations among them.
\end{abstract}

\section{INTRODUCTION}

Holomorphic mappings are very important objects in complex analysis. By using conformal mapping we can map a given domain to a simpler domain on that we know how to solve the problem. Then, by transforming the simpler domain to the given domain we can get the desired answer.

The German mathematician Hermann Amandus Schwarz(1843-1921) worked on conformal mappings and made many fruitful contributions about them. Riemann mapping theorem stated first in 1851 says that every simply connected planar domain except the whole plane can be mapped biholomorphically onto the open unit disc. Riemann proved it by using Dirichlet problem. On the other hand, Schwarz showed the way mapping polygons to the unit circle in 1870. By approximating each simply connected domain by domains bounded by polygons, Schwarz proved the Riemann mapping theorem rigorously (see [16]). The mapping formula is now known as the Schwarz-Christoffel formula, but Schwarz and Christoffel proved it seperately (see $[5])$.

When Schwarz tried to show that the mappings from a convex domain to a series of approximating domains bounded by polygons converge to a limit mapping with the desired properties, he made the Schwarz lemma as a first step in his argument (see [11]). The Schwarz lemma is a simple and highly influential result in the theory of holomorphic mappings. Until now, it bears abundant fruits (see [3], [9], [12], [14]).

Received by the editors May 27, 2011. Revised August 4, 2011. Accepted August 12, 2011. 2000 Mathematics Subject Classification. Primary: 30C80, Secondary: 30C35.

Key words and phrases. Schwarz lemma, boundary fixed point, unit disc, holomorphic map. 
It is interesting that there is another famous mathematician named Schwartz.

The French mathematician Laurent Schwartz(1915-2002) got a Fields Medal in 1950 for his work about distributions. His theory about disribution broadened the differential and integral calculus. It became a powerful tool for application in many areas. Later he worked on stochastic differential calculus. He says that to discover something in mathematics is to overcome an inhibition and a tradition (see [17]).

Note that the first Fields medal was awarded in 1936. So H. Schwarz could't get it. Besides the Schwarz lemma and the Schwarz-Christoffel, we can find his name in many famous theorems such as the Cauchy-Schwarz inequality, the Schwarz reflection principle, and the Schwarzian derivative. Schwarz is often misspelled Schwartz and the Cauchy-Schwarz inequality is often written as the Cauchy-Schwartz inequality by mistake.

In this note, we present the Schwarz lemma and its various representations and study the property of holomorphic functions where fixed points are on the boundary.

\section{The Schwarz Lemma And Its Variations}

At first, the Schwarz lemma appeared without a title in Schwarz's book in 1890 and was named by Carathéodory later in 1912. The proof of the Schwarz lemma given by Schmidt is by using the maximum modulus theorem to the function $f(z) / z$ after redefining the value at $z=0$ where it has a removable singularity as $f^{\prime}(0)$. It became a classical proof of the Schwarz lemma. For the above history of the Schwarz lemma, we refer to [1]. Let us state the Schwarz lemma [10].

Theorem 2.1 (Schwarz Lemma). Let $f$ be a holomorphic function in the open unit disc $U=\{z \in \mathbb{C}:|z|<1\}$ with $f(0)=0$. Suppose that $|f(z)| \leq 1$ in $U$. Then $\left|f^{\prime}(0)\right| \leq 1$ and $|f(z)| \leq|z|$ for all $z \in U$ with equality only for $f(z)=e^{i \alpha} z$, a real.

A variation of the Schwarz lemma is as follows (see [15]).

Corollary 2.2. Let $f$ be a holomorphic function for $|z|<R$ with $f(0)=0$. Suppose that $|f(z)| \leq M$ in $|z|<R$. Then $\left|f^{\prime}(0)\right| \leq M / R$ and $|f(z)| \leq(r / R) M$ for $|z|=$ $r<R$ with equality only for $f(z)=(M / R) e^{i \alpha} z, \alpha$ real.

Application of the Schwarz lemma to a holomorphic function with multiple zeroes at $z=0$ shows the following (see [15]).

Corollary 2.3. Let $f$ be a holomorphic function for $|z|<R$ with $f(0)=f^{\prime}(0)=$ $\cdots=f^{(n-1)}(0)=0$. Suppose that $|f(z)| \leq M$ in $|z|<R$. Then $|f(z)| \leq(r / R)^{n} M$ for all $|z|=r<R$ with equality only for $f(z)=\left(M / R^{n}\right) e^{i \alpha} z^{n}$, $\alpha$ real. 
The above corollary can be proved by writing $f(z)=z^{n} g(z)$ where $g$ is a holomorphic function for $|z|<R$ and using the maximum modulus theorem to $g(z)$.

More generally, the Schwarz lemma can be applied to a function with the information $f\left(z_{0}\right)=w_{0}$ instead of $f(0)=0$. Let the mapping $T_{z_{0}}$ be defined by

$$
T_{z_{0}}(z)=\frac{z_{0}-z}{1-\overline{z_{0}} z}
$$

where $\left|z_{0}\right|<1$. It takes the open unit disc $|z|<1$ onto the open unit disc $|w|<1$ with $T_{z_{0}}\left(z_{0}\right)=0$ and $T_{z_{0}}(0)=z_{0}$. It is interesting that the inverse of $T$ is $T$ itself and $\left|T_{z_{0}}^{\prime}(0)\right|=1-\left|z_{0}\right|^{2}$ and $\left|T_{z_{0}}^{\prime}\left(z_{0}\right)\right|=1 /\left(1-\left|z_{0}\right|^{2}\right)$. Let $f$ be a holomorphic function for $|z|<1$ with $|f(z)| \leq 1$ for $|z|<1$ and $f\left(z_{0}\right)=w_{0}$. Let $F=T_{f\left(z_{0}\right)} \circ f \circ T_{z_{0}}$. Then $F$ satisfies the condition for the Schwarz lemma and so we can get the following variation of the Schwarz lemma called the Schwarz-Pick lemma (see [6], [16]).

Corollary 2.4. Let $f$ be a holomorphic function for $|z|<1$ with $f\left(z_{0}\right)=w_{0}$. Suppose that $|f(z)| \leq 1$ in $|z|<1$. Then

$$
\left|f^{\prime}\left(z_{0}\right)\right| \leq \frac{1-\left|w_{0}\right|^{2}}{1-\left|z_{0}\right|^{2}}
$$

and

$$
\left|T_{w_{0}} \circ f(z)\right| \leq\left|T_{z_{0}}(z)\right|
$$

for $|z|<1$.

If $f$ in Corollary 2.4 fixes $z_{0}$, then $\left|f^{\prime}\left(z_{0}\right)\right| \leq 1$. Note that the equality in the above corollary holds only for Möbius transformation mapping the open unit disc into itself.

The Schwarz-Pick lemma can be generalized to a holomorphic function from the half plane into itself. Let the mapping $H_{z_{0}}$ be defined by

$$
H_{z_{0}}(z)=\frac{z-z_{0}}{z-\overline{z_{0}}}
$$

where $\operatorname{Im} z_{0}>0$. It takes the upper half plane $\operatorname{Im} z>0$ onto the open unit disc $|w|<1$ with $H_{z_{0}}\left(z_{0}\right)=0$ and $\left|H_{z_{0}}^{\prime}\left(z_{0}\right)\right|=1 /\left(2 \operatorname{Im} z_{0}\right)$. The inverse function of $H_{z_{0}}$ is given by

$$
H_{z_{0}}^{-1}(w)=\frac{z_{0}-\overline{z_{0}} w}{1-w}
$$

Let $f$ be a holomorphic function for the upper half plane $\operatorname{Im} z>0$ with $\operatorname{Im} f(z) \geq 0$ in $\operatorname{Im} z>0$. and let $F=H_{f\left(z_{0}\right)} \circ f \circ H_{z_{0}}^{-1}$. Then $F$ satisfies the condition for the Schwarz lemma and the following version of the Schwarz-Pick lemma for the upper half plane was shown by Julia (see [1]). 
Corollary 2.5. Let $f$ be a holomorphic function for the upper half plane Imz $>0$ with $\operatorname{Imf}(z) \geq 0$ in $\operatorname{Imz}>0$ and $z_{0}$ be an arbitrary point in the upper half plane. Then

$$
\left|f^{\prime}\left(z_{0}\right)\right| \leq \frac{\operatorname{Im} f\left(z_{0}\right)}{\operatorname{Im} z_{0}}
$$

for $\operatorname{Im} z_{0}>0$ and the inequality

$$
\left|H_{f\left(z_{0}\right)} \circ f(z)\right| \leq\left|H_{z_{0}}(z)\right|
$$

holds for all $z$ in the upper half plane.

If $f$ in Corollary 2.5 fixes $z_{0}$, then $\left|f^{\prime}\left(z_{0}\right)\right| \leq 1$. Note that the equality in the above corollary holds only for Möbius transformation mapping the upper half plane into itself. When images of the two points are known, Mercer [9] got a sharpened version of the Schwarz-Pick lemma.

The Schwarz lemma is like a stem of a big tree and it can branch out in many directions. For example, the Schwarz lemma can be related with hyperbolic geometry in a way that Corollary 2.4 means $\rho\left(f\left(z_{1}\right), f\left(z_{2}\right)\right) \leq \rho\left(z_{1}, z_{2}\right)$ for all $z_{1}, z_{2}$ in the open unit disc where

$$
\rho\left(z_{1}, z_{2}\right)=\tanh ^{-1}\left|\frac{z_{1}-z_{2}}{1-\bar{z}_{1} z_{2}}\right|
$$

is the distance of $z_{1}$ and $z_{2}$ in the hyperbolic metric in the unit disc (see [11]). In this direction, Bonk and Eremenko [2] assert that for each circular disc on the sphere, the image of every meromorphic map in the plane contains an unbranched disc of at least that size.

Also, the Schwarz lemma can be used to construct the extremal function even for multiply connected domains. Let $z_{0}$ be given in a multiply connected domain. For a fixed point $z$ in the given domain, the extremal functions that have the maximal value of $|f(z)|$ among the functions $f$ mapping a given domain onto the unit disc with $f\left(z_{0}\right)=0$ are proper holomorphic mappings of degree $n$ where $n$ is the connectivity of the given domain (see [14]).

Furthermore, a boundary version of the Schwarz lemma can be extended to smoothly bounded strongly pseudoconvex domains in $\mathbb{C}^{n}$ (see [3]).

\section{Boundary Fixed Points on the Unit Disc}

When equality holds in the Schwarz lemma, a holomorphic self map of the open unit disc must be the identity map if it has another fixed point in the open unit disc except the origin. But it is possible to have another fixed point on the boundary 
of the unit disc for nontrivial holomorphic self map of the open unit disc which has origin as a fixed point. Of course, it should be defined on the boundary or at least at the boundary fixed point. For example, the mapping $f$ from the unit disc onto itself given by $f(z)=z^{5}$ takes 0 to 0 and fixes the boundary points \pm 1 and $\pm i$.

Now, we concern the boundary fixed points of holomorphic mappings of the closed unit disc into itself.

Jack [7] proved Theorem 3.1 by using the derivative of the logarithmic function related to the given function. Note that an extended version of Theorem 3.1 was proved by Ruscheweyh [13] and he mentioned that it is an immediate consequence of Julia's theorem [8].

Theorem 3.1. If a holomorphic function $f$ which is not identically zero maps the closed unit disc $\{z \in \mathbb{C}:|z| \leq 1\}$ into itself with $f(0)=0$, and $|f|$ has a maximum value at $z_{0}$ with $\left|z_{0}\right|=1$, then $z_{0} f^{\prime}\left(z_{0}\right) / f\left(z_{0}\right)$ is real and $z_{0} f^{\prime}\left(z_{0}\right) / f\left(z_{0}\right) \geq 1$.

If $z_{0}=1$, the above theorem says that $f^{\prime}(1) / f(1) \geq 1$.

Let $F(z)=f\left(z_{0} z\right) / f\left(z_{0}\right)$ where $f$ satisfies the condition of Theorem 3.1. Then $F$ is a holomorphic mapping of the closed unit disc into itself with $F(0)=0$ and $F(1)=1$. The maximum value of $|F|$ is taken at 1 and $F^{\prime}(1)=z_{0} f^{\prime}\left(z_{0}\right) / f\left(z_{0}\right)$ (see [1]). Hence the conclusion of Theorem 3.1 is the same as to say that $F^{\prime}(1) \geq 1$.

Julia's theorem leads to the fact that the inequality

$$
F^{\prime}(1) \frac{|1-z|^{2}}{1-|z|^{2}} \geq \frac{|1-F(z)|^{2}}{1-|F(z)|^{2}}
$$

holds for $|z|<1$ and in particular $F^{\prime}(1) \geq 1$ if $F(0)=0$ (see [13]). Hence it induces the desired result of Theorem 3.1.

The following theorem called the boundary Schwarz lemma can be found in [12].

Theorem 3.2. Let $f$ be a holomorphic function from $|z|<1$ into itself with $f(0)=$ 0 . Suppose that for some $z_{0}$ with $\left|z_{0}\right|=1$, f extends continuously to $z_{0}$ with $\left|f\left(z_{0}\right)\right|=$ 1 and $f^{\prime}\left(z_{0}\right)$ exists. Then

$$
\left|f^{\prime}\left(z_{0}\right)\right| \geq \frac{2}{1+\left|f^{\prime}(0)\right|}
$$

Theorem 3.2 implies that $\left|f^{\prime}\left(z_{0}\right)\right| \geq 1$ and equality holds only for $f(z)=e^{i \alpha} z$, $\alpha$ real. Especially, if $f(0)=f^{\prime}(0)=0$ in the hypothesis of Theorem 3.2, then $\left|f^{\prime}\left(z_{0}\right)\right| \geq 2$ and equality holds only for $f(z)=e^{i \alpha} z^{2}$, $\alpha$ real. Carathéodory [4, pp. 28-32] dealt with the other types of the boundary Schwarz lemma via the angular derivative. 
From now on, we consider the case in which all the fixed points are on the boundary. Boas mentioned that the following theorem in [1] is a corollary of results of Wolff.

Theorem 3.3. If a holomorphic function $f$ which is not identically zero maps the closed unit disc $\{z \in \mathbb{C}:|z| \leq 1\}$ into itself and all the fixed points are on the boundary, then there is a distinguished fixed point $z_{0}$ at which $f^{\prime}\left(z_{0}\right) \leq 1$ and at the other fixed points $\zeta, f^{\prime}(\zeta) f^{\prime}\left(z_{0}\right) \geq 1$.

The following example in [1] illustrates Theorem 3.3.

Example 3.4. Let $f(z)=\left(\frac{z-\frac{2}{3}}{1-\frac{2}{3} z}\right)^{3}$. Then $f$ maps the closed unit disc onto itself and has 4 fixed points $\pm 1, \frac{9 \pm 5 \sqrt{7} i}{16}$ on the boundary of the unit disc. Then, -1 is a distinguished fixed point with $f^{\prime}(-1)=3 / 5$ and

$$
f^{\prime}(1)=15, \quad f^{\prime}\left(\frac{9 \pm 5 \sqrt{7} i}{16}\right)=12 / 5 .
$$

We can construct another example. The Möbius transformation from the unit disc onto the unit disc is the most classical example for Theorem 3.3.

Example 3.5. Let $f(z)=(z-r) /(1-r z)$, where $r$ is real with $|r|<1$. The fixed points of $f$ are \pm 1 . If $0 \leq r<1$, then -1 is a distinguished fixed point with $f^{\prime}(-1)=(1-r) /(1+r) \leq 1$. If $-1<r \leq 0$, then 1 is a distinguished fixed point with $f^{\prime}(1)=(1+r) /(1-r) \leq 1$. In both cases, $f^{\prime}(-1) f^{\prime}(1)=1$.

Now, we generalize the function in Example 3.4 to get a bunch of functions satisfying Theorem 3.3. During the process, we get interesting results about holomorphic functions.

Lemma 3.6. Let $f$ be a holomorphic function on the closed unit disc defined by

$$
f(z)=e^{i \beta}\left(\frac{z-r e^{i \alpha}}{1-r e^{-i \alpha} z}\right)^{3}
$$

where $\alpha, \beta, r$ are real with $|r|<1$. Then $\pm e^{i \alpha}$ are fixed points of $f$ if and only if $\beta=-2 \alpha$.

Proof. If $\pm e^{i \alpha}$ are fixed points of $f$, then $f\left( \pm e^{i \alpha}\right)=e^{i \beta}\left( \pm e^{i \alpha}\right)^{3}= \pm e^{i \alpha}$ implies that $\beta=-2 \alpha$.

If $\beta=-2 \alpha$, then the fixed points of $f$ satisfy the equation

$$
e^{-2 i \alpha}\left(\frac{z-r e^{i \alpha}}{1-r e^{-i \alpha} z}\right)^{3}=z
$$


Hence,

$$
e^{-2 i \alpha}\left(z-r e^{i \alpha}\right)^{3}=z\left(1-r e^{-i \alpha} z\right)^{3}
$$

which is factorized as follows:

$$
\left(z e^{-i \alpha}-1\right)\left(z e^{-i \alpha}+1\right)\left(r^{3} e^{i \alpha}\left(z^{2} e^{-2 i \alpha}+1\right)+z\left(1-3 r^{2}\right)\right)=0 .
$$

So, $\pm e^{i \alpha}$ are fixed points of $f$.

We can explicitly express all the fixed points of the function given in Lemma 3.6 where $\beta=-2 \alpha$ and we find a condition for $r$ so that all the fixed points of the given function are the boundary fixed points.

Lemma 3.7. Let $f$ be a function on the unit disc defined by

$$
f(z)=e^{-2 i \alpha}\left(\frac{z-r e^{i \alpha}}{1-r e^{-i \alpha} z}\right)^{3}
$$

where $\alpha, r$ are real with $|r|<1$. Then all the fixed points of $f$ are on the boundary of the unit disc if and only if $1 / 2 \leq|r|<1$.

Proof. By (3.1), all the fixed points of $f$ are

Let us denote

$$
\pm e^{i \alpha}, \quad \frac{3 r^{2}-1 \pm \sqrt{\left(3 r^{2}-1\right)^{2}-4 r^{6}}}{2 r^{3} e^{-i \alpha}}
$$

$$
z_{1}=\frac{3 r^{2}-1+\sqrt{\left(3 r^{2}-1\right)^{2}-4 r^{6}}}{2 r^{3} e^{-i \alpha}}, \quad z_{2}=\frac{3 r^{2}-1-\sqrt{\left(3 r^{2}-1\right)^{2}-4 r^{6}}}{2 r^{3} e^{-i \alpha}} .
$$

If $1 / 2 \leq|r|<1$, then

$$
\left(3 r^{2}-1\right)^{2}-4 r^{6}=-(1-r)^{2}(1+r)^{2}(2 r-1)(2 r+1) \leq 0 .
$$

Hence,

and similarly $\left|z_{2}\right|=1$.

$$
\left|z_{1}\right|=\left|\frac{3 r^{2}-1+i \sqrt{-\left(3 r^{2}-1\right)^{2}+4 r^{6}}}{2 r^{3} e^{-i \alpha}}\right|=1
$$

If $|r|<1 / 2$, then $\left(3 r^{2}-1\right)^{2}-4 r^{6}>0$. It implies that $\left|z_{1}\right|<1<\left|z_{2}\right|$ by calculation. Hence, the conclusion holds.

At all the boundary fixed points, we compute the derivatives of $f$ to get some relation among them.

Theorem 3.8. Let $f$ be as in the hypothesis of Lemma 3.7 and let $1 / 2 \leq|r|<1$. If $1 / 2 \leq r<1$, then $-e^{i \alpha}$ is a distinguished fixed point with $f^{\prime}\left(-e^{i \alpha}\right) \leq 1$ and at the other fixed points $\zeta, f^{\prime}(\zeta) f^{\prime}\left(-e^{i \alpha}\right) \geq 1$. 
If $-1<r \leq-1 / 2$, then $e^{i \alpha}$ is a distinguished fixed point with $f^{\prime}\left(e^{i \alpha}\right) \leq 1$ and at the other fixed points $\zeta, f^{\prime}(\zeta) f^{\prime}\left(e^{i \alpha}\right) \geq 1$.

Proof. By calculation,

$$
f^{\prime}(z)=3\left(1-r^{2}\right) f(z) /\left(z-r e^{i \alpha}\right)\left(1-r e^{-i \alpha} z\right) .
$$

Hence,

$$
f^{\prime}\left(-e^{i \alpha}\right)=3(1-r) /(1+r), \quad f^{\prime}\left(e^{i \alpha}\right)=3(1+r) /(1-r)
$$

and

$$
f^{\prime}\left(z_{1}\right)=f^{\prime}\left(z_{2}\right)=3 r^{2} /\left(1-r^{2}\right)
$$

where $z_{1}$ and $z_{2}$ are fixed points of $f$ except $\pm e^{i \alpha}$.

Assume $1 / 2 \leq r<1$. The derivatives of $f$ satisfy that

$$
f^{\prime}\left(-e^{i \alpha}\right) \leq 1, \quad f^{\prime}\left(-e^{i \alpha}\right) f^{\prime}\left(e^{i \alpha}\right)=9
$$

and

$$
f^{\prime}\left(z_{1}\right) f^{\prime}\left(-e^{i \alpha}\right)=f^{\prime}\left(z_{2}\right) f^{\prime}\left(-e^{i \alpha}\right)=9 r^{2} /(1+r)^{2} \geq 1
$$

since $9 r^{2}-(1+r)^{2} \geq 0$ if $r \geq 1 / 2$.

If $-1<r \leq-1 / 2$, similarly we get the desired conclusion.

We can construct many functions having fixed points only on the boundary with given inequalities relating their derivatives by Theorem 3.8. We make an example as a variation of Example 3.4.

Example 3.9. Let $f(z)=-\left(\frac{z-\frac{2}{3} i}{1+\frac{2}{3} i z}\right)^{3}$. Then $f$ has fixed points $\pm i, \frac{9 i \pm 5 \sqrt{7}}{16}$. A distinguished fixed point is $-i$ with $f^{\prime}(-i)=3 / 5$ and $f^{\prime}(i)=15, f^{\prime}\left(\frac{9 i \pm 5 \sqrt{7}}{16}\right)=12 / 5$. Hence this $f$ satisfies the desired conclusion in Theorem 3.8.

Remark 3.10. If $r=1 / 2$ in (3.2), there are only 2 fixed points $\pm e^{i \alpha}$ of $f$ in (3.2) on the boundary and $f^{\prime}\left(-e^{i \alpha}\right)=1, f^{\prime}\left(e^{i \alpha}\right)=9$.

We can give another type of functions by simply changing the exponent 3 of the function $f$ in Lemma 3.6 into 2. A similar argument given in Lemma 3.6 will establish the following lemma.

Lemma 3.11. Let

$$
f(z)=e^{i \beta}\left(\frac{z-e^{i \alpha} r}{1-e^{-i \alpha} r z}\right)^{2}
$$

where $\alpha, \beta, r$ are real with $|r|<1$. Then $e^{i \alpha}$ is a fixed point of $f$ if and only if $\beta=-\alpha$. 
Finally, we find a condition for $f$ given in Lemma 3.11 where $\beta=-\alpha$ to have fixed points only on the boundary with given inequalities of its derivatives.

Theorem 3.12. Assume that

$$
f(z)=e^{-i \alpha}\left(\frac{z-e^{i \alpha} r}{1-e^{-i \alpha} r z}\right)^{2}
$$

where $\alpha, r$ are real with $-1<r \leq-1 / 3$. Then $e^{i \alpha}$ is a distinguished fixed point with $f^{\prime}\left(e^{i \alpha}\right) \leq 1$ and at the other fixed points $\zeta, f^{\prime}(\zeta) f^{\prime}\left(e^{i \alpha}\right) \geq 1$.

Proof. The equation $f(z)=z$ implies that

$$
e^{-i \alpha}\left(z-e^{i \alpha} r\right)^{2}=z\left(1-e^{-i \alpha} r z\right)^{2}
$$

is factorized into

$$
\left(z e^{-i \alpha}-1\right)\left(r^{2} e^{-i \alpha} z^{2}+\left(r^{2}-2 r-1\right) z+r^{2} e^{i \alpha}\right)=0 .
$$

Hence, the fixed points of $f$ are

$$
e^{i \alpha}, \frac{1+2 r-r^{2} \pm \sqrt{\left(r^{2}-2 r-1\right)^{2}-4 r^{4}}}{2 r^{2} e^{-i \alpha}} .
$$

Let us denote

$$
z_{1}=\frac{1+2 r-r^{2}+\sqrt{\left(r^{2}-2 r-1\right)^{2}-4 r^{4}}}{2 r^{2} e^{-i \alpha}}
$$

and

$$
z_{2}=\frac{1+2 r-r^{2}-\sqrt{\left(r^{2}-2 r-1\right)^{2}-4 r^{4}}}{2 r^{2} e^{-i \alpha}} .
$$

If $-1<r \leq-1 / 3$, then

$$
\left(r^{2}-2 r-1\right)^{2}-4 r^{4}=(1+r)^{2}(1-r)(3 r+1) \leq 0 .
$$

Hence $z_{1}$ and $z_{2}$ are on the boundary of the unit disc with $f^{\prime}\left(z_{1}\right)=f^{\prime}\left(z_{2}\right)=-2 r /(1+$ $r)$. Therefore $e^{i \alpha}$ is a distinguished fixed point of $f$ with $f^{\prime}\left(e^{i \alpha}\right)=2(1+r) /(1-r) \leq 1$ and $f^{\prime}\left(e^{i \alpha}\right) f^{\prime}\left(z_{1}\right)=f^{\prime}\left(e^{i \alpha}\right) f^{\prime}\left(z_{2}\right)=-4 r /(1-r) \geq 1$ when $-1<r \leq-1 / 3$.

Remark 3.13. If $r=-1 / 3$ in (3.3), there is only one fixed point $e^{i \alpha}$ of $f$ in (3.3) on the boundary and $f^{\prime}\left(e^{i \alpha}\right)=1$.

\section{REFERENCES}

1. H.P. Boas: Julius and Julia: Mastering the art of the Schwarz lemma. Amer. Math. Monthly 117 (2010), 770-785. 
2. M. Bonk \& A. Eremenko: Covering properties of meromorphic functions, negative curvature and spherical geometry. Ann. Math. 152 (2000), 551-592.

3. D. Burns \& S. Krantz: Rigidity of holomorphic mappings and a new Schwarz lemma at the boundary. J. Amer. Math. Soc. 7 (1994), 661-676.

4. C. Carathéodory: Theory of Functions of a Complex Variable. Vol 2, Chelsea, New York, 1954.

5. J. Cases: Breakthrough in conformal mapping. SIAM News 41 (2008), no. 1, http://www.siam.org/pdf/news/1297.pdf.

6. R. Greene \& S. Krantz: Function theory of one complex variable. Graduate studies in Mathematics Vol 40, Amer. Math. Soc., Providence, 2002.

7. S. Jack: Functions starlike and convex of order $\alpha$. J. London Math. Soc. 3 (1971), 469-474.

8. G. Julia: Extension nouvelle d'un Lemma de Schwarz. Acta Math. 42 (1920), 349-355.

9. P. R. Mercer: Sharpened versions of the Schwarz lemma. J. Math. Anal. Appl. 205 (1997), 508-511.

10. Z. Nehari: Conformal Mapping. Dover publications, Inc., New York, 1952.

11. R. Osserman: From Schwarz to Pick to Ahlfors and Beyond. Notices Amer. Math. Soc. 46 (1999), 868-873.

12. __ A Sharp Schwarz inequality on the boundary. Proc. Amer. Math. Soc. 128 (2000), 3513-3517.

13. S. Ruscheweyh: Neighborhoods of univalent functions. Proc. Amer. Math. Soc. 81 (1981), 521-527.

14. G. Schmieder: On Schwarz's Lemma in multiply connected domains. Comput. Methods Funct. Theory 5 (2005), 159-184.

15. H. Silverman: Complex variables. Houghton Mifflin, 1975.

16. http://en.wikipedia.org/wiki/Schwarz_lemma.

17. http://www-history.mcs.st-and.ac.uk.

Department of Mathematics, University of Suwon, Gyeonggi-do 445-743, Korea

Email address: mjeong@suwon.ac.kr 